\title{
Vom Leben im Heim
}

\section{Modelle zur Reflexion beruflicher Praxis in der Altenpflege}

\author{
ULRIKE HUBER \\ Ulrike Huber ist Heimleiterin von \\ "Haus St. Katharina" in Endingen in \\ Baden-Württemberg, eine vollsta- \\ tionäre Einrichtung der Altenhilfe \\ mit 132 Plätzen und eine Einrichtung \\ des Saarländischen Schwesternver- \\ bandes. Nach ihrer Ausbildung zur \\ Krankenschwester studierte Ulrike \\ Huber berufsbegleitend Pflegema- \\ nagement und hat dann noch ein \\ Masterstudium in Supervision abge- \\ schlossen. Sie hat langjährige Be- \\ rufs- und Leitungserfahrung und ist \\ nebenberuflich als Honorardozentin \\ im Fort- und Weiterbildungsbereich \\ sowie als Supervisorin tätig. \\ www.schwesternverband.de
}

\author{
Stationäre Pflegeheime stehen in der Kritik von \\ Öffentlichkeit und Experten. Viele alte Menschen \\ kommen erst in eine solche Einrichtung, "wenn \\ es gar nicht mehr anders geht", bei Demenz, nach \\ einem Sturz oder nach einem Schlaganfall. Um das \\ "System Pflegeheim" zu verstehen und zu verändern, \\ können drei Theoriemodelle gute Dienste leisten.
}

Das Arbeitsfeld der Altenhilfe entwickelt sich ständig weiter. Anhand der Verknüpfung von drei theoretischen Modellen kann eine Reflexion der beruflichen Praxis in der stationären Altenpflege erfolgen. Diese Reflexion auf verschiedenen Erklärungsebenen will für »typische « Phänomene sensibilisieren sowie Entwicklungsperspektiven und Handlungsfelder aufzeigen.

\section{Erste Erklärungsebene: Das Vier-Felder-Modell}

Das Vier-Felder-Modell nach Prof. Dr. Frank Schulz-Nieswandt ist ein Sektorenmodell, das die Bedingungen darstellt, um Wohlfahrt im Alter sicherzustellen. Dieses Modell wurde ursprünglich in der sozialpolitischen Diskussion um Versorgungsstrukturen verwendet. Es eignet sich aber auch als Reflexionsmodell beruflicher Pflegepraxis, weil sich auf allen vier Feldern immer wieder neue Veränderungen und Entwicklungen ergeben, die die Pflege beeinflussen. Die vier Felder nennt Schulz-Nieswandt primäre Netze, Staat, Markt und intermediärer Bereich.

- Primäre Netze: Die primären sozialen Netze eines pflegebedürftigen Menschen sind Familie, Freunde, Nachbarn etc. Diese Netze sind einem sozialen Wandel unterworfen: Es gibt immer mehr unvollständige Familien, der Anteil berufstätiger Frauen hat in den letzten Jahrzehnten deutlich zugenommen und es bestehen immer größere und flexiblere
Mobilitätsanforderungen. Außerdem ist die Zahl von Ein- und Zweipersonenhaushalten gestiegen und macht inzwischen zwei Drittel aller Haushalte aus, so dass dieses "primäre Pflegepotenzial « immer weniger zur Verfügung steht und es für Angehörige immer schwieriger wird, Pflege zu leisten. Dieser soziale Wandel hat zur Einführung der Pflegeversicherung geführt und wir kennen die beschriebenen Phänomene gut aus unseren Heimaufnahmegesprächen.

- Staat: Der Staat beeinflusst die Pflege (und das Marktgeschehen) durch Gesetze und Verordnungen wie das Heimgesetz, die Landesheimpersonalverordnung oder die Landesheimbauverordnung. Aber auch volkswirtschaftliche Entscheidungen wie Versorgungs- oder Sicherstellungsaufträge, die Einführung der Pflegeversicherung oder die Anwendung des Subsidiaritätsprinzips zur Finanzierung von Pflegeleistungen wirken auf die Pflege ein. Hinter diesen volkswirtschaftlichen Entscheidungen steht letztlich die Frage, wie viel einer Gesellschaft die Sicherstellung von Pflege wert ist.

- Markt: Der Markt umfasst Pflegeleistungen durch ambulante Dienste bis hin zur vollstationären Pflege. Er wird wie jeder Markt durch Angebot und Nachfrage reguliert. Derzeit besteht in den meisten Regionen ein Überangebot an stationären Pflegeplätzen und das Marktgeschehen wird damit zu einem Verdrängungswettbewerb. Gleichzeitig stehen die 
stationären Pflegeeinrichtungen in der öffentlichen Meinung in der Kritik - in den Medien überwiegt die negative Berichterstattung deutlich. Doch solange die öffentliche Diskussion in dieser Art und Weise geführt wird, dürfte es schwierig sein, das Image der Pflegeberufe aufzuwerten und dem Fachkräftemangel zu begegnen. In der Entscheidung, welches Pflegeangebot nachgefragt wird, steckt auch die Frage, ob man
Wohngemeinschaften. Diese binden primäre Netze (Angehörige, Freunde) und Ehrenamt als konstituierende Elemente ein. Es ist also nicht mehr so, dass die Vernetzung der beschriebenen vier Felder als wünschenswerte Idealform erfolgt, sondern die Vernetzung ist zwingend notwendig, damit das Versorgungsmodell überhaupt funktioniert. Von der baden-württembergischen Landesregierung werden solche Projekte gemäß dem Grundsatz »ambulant

\section{"Zu entscheiden ist die Frage, ob man die Hilfe zum Menschen bringt oder den Menschen zur Hilfe»}

die Hilfe zum Menschen bringt oder den Menschen zur Hilfe. Pflegebedürftige Menschen in ein Pflegeheim zu bringen, hat nicht gerade ein hohes Sozialprestige. Immer noch sind für die Angehörigen mit dieser Entscheidung häufig Schuldgefühle verbunden. Die Menschen kommen erst in eine Einrichtung, wenn es gar nicht mehr anders geht oder wenn ein Krisenereignis wie ein Sturz mit Fraktur oder ein Schlaganfall eingetreten ist. So sind die Bewohner, die in ein Pflegeheim einziehen, immer älter und oft kränker.

- Intermediärer Bereich: Es wird immer gesagt - und auch im Gesetzestext der Pflegeversicherung (SGB XI) ausdrücklich formuliert -, dass der intermediäre Bereich, der Bereich des Ehrenamts und bürgerschaftlichen Engagements immer wichtiger wird, weil vollumfängliche Pflege von den Familien nicht mehr leistbar ist, volkswirtschaftlich nicht finanzierbar und allein über Institutionen nicht bezahlbar ist. Aber wie groß ist die Ressource Ehrenamt wirklich? Es gibt viele, die bereit sind, gelegentlich was zu tun - aber nur wenige, die sich verbindlich und verlässlich festlegen. Kann und darf Ehrenamt tatsächlich ein konstituierendes Element in der Versorgung Pflegebedürftiger sein oder sollte es nicht vielmehr ein zusätzliches Element sein?

Es entstehen derzeit auf dem »Pflegemarkt« verschiedene Modellprojekte $\mathrm{zu}$ alternativen Wohnformen und vor stationär« ausdrücklich unterstützt. Auch wenn diese Modellprojekte mit gutem Erfolg arbeiten, bleibt doch die Frage offen, ob die Ressourcen aus den primären Netzen und bürgerschaftlichem Engagement ausreichen, um über (teilweise hochsubventionierte) Modellprojekte hinaus eine flächendeckende Versorgung sicherzustellen.

\section{Zweite Erklärungsebene: Die Theorie der totalen Institution}

Die zweite Ebene des Reflexionsmodells ist die Theorie der totalen Institution von Erving Goffman. Der amerikanische Soziologe Erving Goffman wurde in der Vergangenheit oft mit seiner Kritik der totalen Institution mit der stationären Pflege in Verbindung gebracht. »Total « soll heißen: Ein Mensch verbringt seine gesamte Zeit an einem bestimmten Ort. Zu totalen Institutionen zählt der Soziologe unter anderem Gefängnisse und Psychiatrien, aber auch Pflegeheime.

Goffman beschrieb in den 1970er Jahren Kennzeichen totaler Institutionen, die bis heute Anwendung finden. Einige dieser Kennzeichen sind:

- Alle Angelegenheiten des Lebens finden an ein und derselben Stelle, unter ein und derselben Autorität statt.

- Die Phasen des Tages sind exakt geplant, es gelten explizite formale Regeln.

- Die Definitionsmacht über den Tagesablauf liegt beim Personal.

- Es besteht eine Trennung zwischen den Bewohnern, die in der Institution leben, und den Mitarbeitenden, die in der Institution arbeiten. Die Bewohner sind in der Möglichkeit, soziale Kontakte wahrzunehmen, beschränkt. Die Mitarbeitenden verlassen die Einrichtung nach Dienstende und sind sozial integriert. Damit sind totale Institutionen "Zwitter zwischen Wohn- und Lebensgemeinschaft einerseits und formaler Organisation andererseits.

Das Modell von Goffman ist zunächst rein deskriptiv. Ein negativer Inhalt ergibt sich erst aus den negativen Folgen des Lebens in einer totalen Institution, nämlich der Erzwingung von Anpassung. Bei einem ehrlichen und selbstkritischen Umgang mit der Situation in der stationären Pflege muss man einräumen, dass diese Theorie immer noch eine Relevanz hat, wie die Praxis zeigt (vgl. Kasten mit Praxisbeispiel Seite 13).

Ein weiteres Merkmal totaler Institutionen ist, dass der Eintritt in eine totale Institution in der Regel nicht freiwillig erfolgt. Auch dies trifft für Pflegeheime teilweise zu. Es kommt selten jemand aus Überzeugung, sondern eher mangels Alternativen, oft nach gesundheitlichen Krisenereignissen wie Schlaganfall, Sturz mit Fraktur oder Demenz. Somit besteht ein Zwangscharakter.

Man darf aber auch nicht vergessen, dass Pflege in der eigenen Häuslichkeit auch Zwangssysteme sein können. Ich muss als Pflegebedürftiger um Hilfe bitten und ich muss als Angehöriger pflegen. Und so kann eine Institution hier auch eine Entlastung bringen und letztlich zur Stabilisierung der familiären Beziehungen beitragen, weil Überforderungssituationen durchbrochen werden.

Eine Beschränkung der sozialen Kontakte besteht oft auch zuhause, beispielsweise wenn der Allgemeinzustand der Pflegebedürftigen die Wahrnehmung von sozialen Kontakten beeinträchtigt oder wenn Außenkontakte deshalb nicht mehr möglich sind, weil die Privatwohnung im zweiten Obergeschoss liegt und die Treppe ein unüberwindliches Hindernis darstellt. In diesen Fällen erleben wir in der Pflegeeinrichtung, dass pflegebedürftige ältere Menschen wieder aktiver und kommunikativer werden, weil sie mehr Möglichkeiten haben, am sozialen Ge- 
schehen innerhalb und außerhalb des Pflegeheims teilzunehmen.

Aus dieser Institutionskritik entstanden in den letzten Jahren verschiedene Suchbewegungen nach Alternativen, beispielsweise die verschiedenen Generationen der KDA-Hausgemeinschaftsmodelle. Oder die Ambulantisierung im Heim, das heißt, die Organisation der Pflege im Heim nach Tourenmodellen, ähnlich wie die eines ambulanten Pflegedienstes. Außerdem sind alternative Wohnformen wie Wohngemeinschaften für Menschen mit Demenz entstanden.

In diesen Modellen wird versucht, die Selbstbestimmung der Bewohner zu stärken und Hauswirtschaft und Betreuung der Pflege voranzustellen, ausgehend von der Idee, dass ein Mensch zwar 24 Stunden am Tag wohnt und seine Zeit verbringt, nicht aber 24 Stunden Pflege benötigt. Beschäftigung wird möglichst alltagsnah organisiert und nicht als »künstliches « Angebot.

Doch auch in diesen Einrichtungen werden Institutionalisierungseffekte beschrieben. Institutionalisierung ist das, was offensichtlich automatisch geschieht, wenn man Menschen aus dem häuslichen Umfeld herausnimmt und ihren Lebensmittelpunkt in eine Institution verlagert. Wenn man etwas anderes will, muss man mit viel Energie und Aufmerksamkeit den Effekten der Institutionalisierung entgegenwirken, sowohl durch entsprechende organisatorische Konzepte als auch durch die konsequente Öffnung der Einrichtung und nicht zuletzt durch eine ständig kritische Reflexion von der Haltung und Einstellung der Mitarbeitenden.

\section{Dritte Erklärungsebene: Die Theorie der Beschleunigung}

Der Soziologe Hartmut Rosa hat im Jahre 2005 eine Theorie des Umgangs mit der Zeit und dem Zeiterleben entwickelt und nennt sie die »Theorie der Beschleunigung des Zeiterlebens und des Zeitbewusstseins «. Sie bildet die dritte Ebene des beschriebenen Reflexionsmodells. Rosa unterscheidet vier Dimensionen:

- Das occasionale Zeitbewusstsein: Hier wird lediglich zwischen "jetzt « und »nicht-jetzt « unterschieden.
- Das zyklische Zeitbewusstsein unterscheidet zwischen vorher und nachher.

- Das lineare Zeitbewusstsein ist gekennzeichnet durch Kalender, Uhren, Zeiterfassung ... Es drückt die Zeitachse von der Vergangenheit durch die Gegenwart in die Zukunft aus.

- Das Zeitbewusstsein der Beschleunigung - das ist ein Phänomen der Moderne. Es ist Ausdruck für ein immer schnelleres Lebenstempo, mit dem eine Gesellschaft sich organisiert. Beschleunigung heißt mehr Handlung, mehr Tun, mehr Komplexität und mehr Dichte, immer schnellere Veränderungen, immer weniger Beständigkeit und Nachhaltigkeit, eine Zunahme von Anforderungen und damit verbunden auch eine Zunah-

\section{Praxisbeispiel: »lch gehe ins Bett, wenn Sie es sagen«}

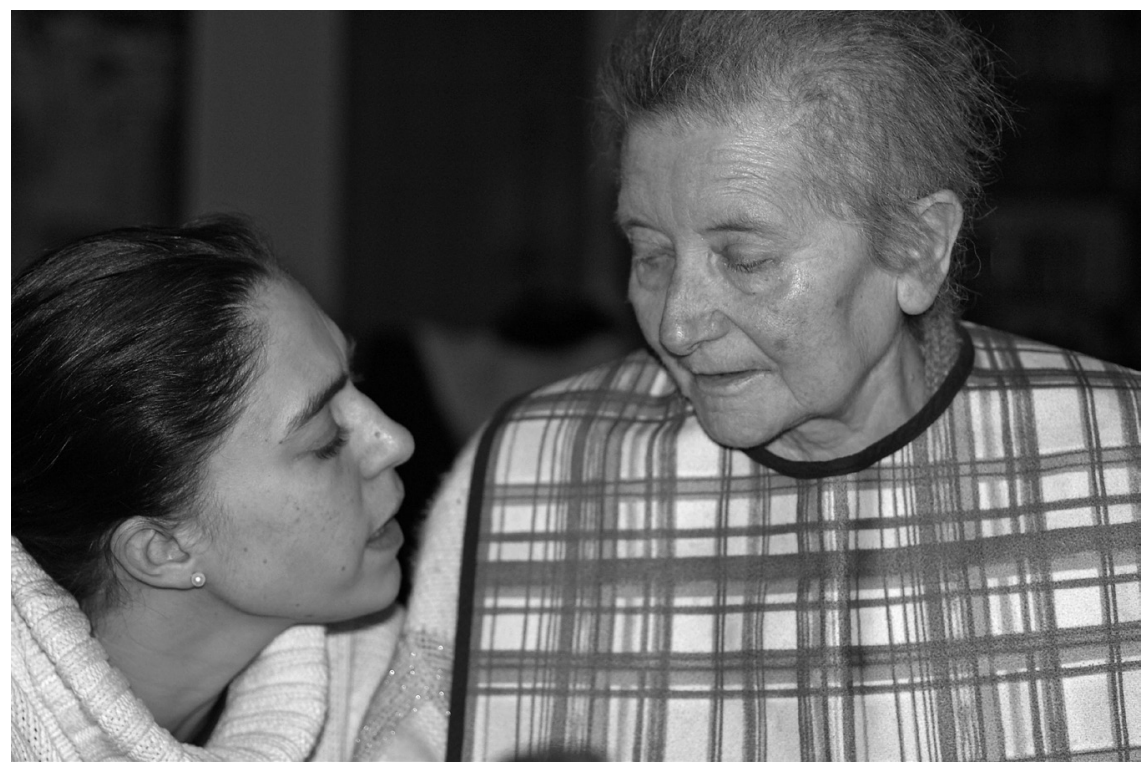

Die Theorie der totalen Institution von Erving Goffman beschreibt Einrichtungen wie Gefängnisse und Pflegeheime zunächst rein sachlich. Das Problematische an der totalen Institution ergibt sich aus den negativen Folgen des Lebens in einer solchen Einrichtung, nämlich der Erzwingung von Anpassung. Ich möchte dies an einem Beispiel aus dem von mir geleiteten "Haus St. Katharina" zeigen In einem Einzugsgespräch erfrage ich den bisherigen Tagesablauf der neuen Bewohner und erfahre so vieles über Vorlieben und Gewohnheiten. Nun wechselte eine Bewohnerin aus einer anderen Pflegeeinrichtung zu uns. Auf die Frage, wann me der Gleichzeitigkeit (und Widersprüchlichkeit) von Anforderungen.

Prof. Dr. Katharina Gröning hat in eigener Auseinandersetzung mit diesem Modell eine fünfte Dimension hinzugefügt: das sakrale Zeitbewusstsein. Damit ist die Phase gemeint, wenn man aus der Zeit geht, also der Sterbeprozess.

Im Alltag einer Pflegeeinrichtung prallen diese Zeitebenen alle aufeinander und sind in einer Gleichzeitigkeit vorhanden wie kaum an einem anderen Ort. Beispielsweise ist das dominante Zeitbewusstsein von Demenzkranken das occassionale, Mitarbeitende sind hingegen dem Zeitbewusstsein der Beschleunigung unterworfen. Ein Beobachter im Altenheim kann sehr schnell einen Eindruck für das Aufeinanderprallen unterschiedlicher Zeitbewusst- 
seinsebenen bekommen, wenn er sieht, wie gehetzte Mitarbeitende an den sich langsam an den Gängen entlang tastenden Bewohnerinnen vorbeieilen. Das bedeutet, für die Arbeit im Pflegeheim besteht die besondere Anforderung, mit den großen Zeitspannungen zwischen der Lebenswelt und dem Zeitbewusstsein der Bewohner und der Zeitkultur der Organisation zu vermitteln.

Zeitmangel in der Pflege ist nicht nur deshalb da, weil sich objektiv die Anforderungen tatsächlich ständig erhöhen, sondern er begründet sich auch in dieser Spannung zwischen den verschiedenen Zeitkulturen und wird so doppelt spürbar. Zu dieser Beschleunigung haben zum Beispiel die Konstruktion des Pflegeversicherungsgesetzes, der Pflegebegriff und die Definition des Pflegeprozesses, heimrechtliche Bestimmungen, externe und interne Qualitätsprüfungen oder Kostendruck beigetragen. Aber auch die fortschreitende Professionalisierung in der Altenpflege und die Veränderung des Altersbildes vom Defizitmodell zum Kompetenzmodell. Dadurch sind gute ganzheitliche und nichtfunktionale Pflegekonzepte entstanden. Gleichzeitig bestimmt der Kostendruck die Umsetzung dieser Pflegekonzepte und damit werden die Konzepte selbst wieder zur Ressourcenfrage.

Das Zeitbewusstsein der Organisation - das der Beschleunigung - wird dem occasionalen und kreishaften Zeitbewusstsein der Bewohner in der Regel dominant vorangestellt und übergeordnet, von den Bewohnern wird es aber als fremd und unpassend wahrgenommen. Dies wiederum begünstigt die Institutionalisierungseffekte. Deshalb bedarf es einer »Zeitsynchronisation «. Ziel muss es sein, im überwiegend occasionalen Zeitbewusstsein der Bewohner Lebensqualität im Hier und Jetzt zu schaffen und möglichst viele gute Momente aneinanderzureihen.

\section{Perspektiven und Handlungsfelder}

Aus den vorgestellten Erklärungsmodellen ergeben sich Anforderungen an die strategische Ausrichtung der Pflegeheime: Welche baulichen Möglichkeiten lassen welche Konzeption überhaupt zu? Ist es nun angesagt, auf die »modernen« Konzeptionen wie
Hausgemeinschaftsmodelle und Ambulantisierung umzusteigen? Oder gibt es in den konventionellen Pflegeheimen auch Stärken, die erhaltenswert sind? Welche Konzeption passt zu welchen Bewohnern? Und wie lange passt diese Konzeption im Hinblick darauf, dass der Allgemeinzustand des Heimbewohners sich im Laufe des Aufenthalts weiter verändert? Kann man mit einem wie auch immer gearteten Konzept, gerade unter dem Anspruch der Berücksichtigung von Individualität, tatsächlich einer heterogenen Klientenstruktur gerecht werden? Oder muss es haus- oder trägerintern nicht auch eine Heterogenität von Konzeptionen geben?

\section{Literatur}

Goffman, Erving: Asyle. Über die soziale Situation psychiatrischer Patienten und anderer Insassen. Frankfurt am Main 1973, Suhrkamp Verlag.

Gröning, Katharina: Zeitantagonismus in der Altenhilfe und die Bedeutung der Supervision. In: Gesundheitsförderung und Supervision, Forum Supervision, Heft 34/2009 S. 47-58, Fachhochschulverlag Frankfurt am Main.

Rosa, Hartmut: Beschleunigung. Die Veränderung der Zeitstruktur in der Moderne. Frankfurt am Main 2005, Suhrkamp Verlag Schulz-Nieswandt: unveröffentlichte Seminarunterlagen.

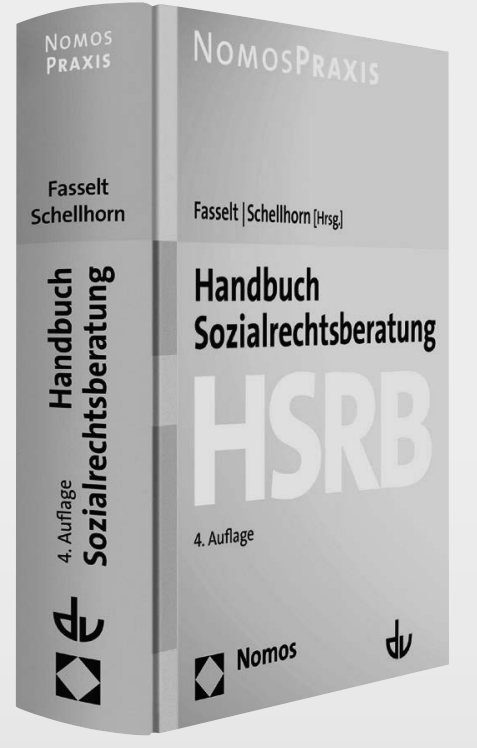

\section{Handbuch}

\section{Sozialrechtsberatung - HSRB}

Herausgegeben von

Prof. Dr. Ursula Fasselt und

Prof. Dr. Helmut Schellhorn

4. Auflage 2012, 884 S., geb.,

inkl. Plakat und einjähriger

Onlinenutzung, 98,- $€$

ISBN 978-3-8329-7737-5

Wer aktuell und kompetent beraten will, kommt an dem Handbuch für die Sozialrechtsberatung nicht vorbei: Alle Lebenslagen, die in der Beratungspraxis typischerweise vorkommen, werden auf I hre Anspruchsgrundlagen zurückgeführt. Sie erkennen, welche Leistungen dem Betroffenen zustehen, wer zuständig ist und wie es um die Durchsetzung bestellt ist.

NEU: Im Preis inbegriffen ist die online-Version mit unterjähriger Aktualisierung und Verlinkung auf die zitierten Gesetze und Urteile im Volltext.

"Dieses Handbuch darf auf den Schreibtischen von Beraterinnen und Beratern, die in ihrem Beratungsalltag mit Sozialrechtsproblemen zu tun haben, nicht fehlen." Friedrich Maus, Forum Sozial 4/11, zur Vorauflage

Weitere Informationen: www.nomos-shop.de/19473 